THE ASTROPHYSICAL JOURNAL SUPPLEMENT SERIES, 90:797-806, 1994 February

(C) 1994. The American Astronomical Society. All rights reserved. Printed in U.S.A.

\title{
RELATIVISTIC PARTICLE ACCELERATION IN PLERIONS
}

\author{
JONATHAN ARONS ${ }^{1}$ \\ University of California \\ AND \\ MARCO TAVANI ${ }^{2}$ \\ Physics Department, Princeton University \\ Received 1993 March 1; accepted 1993 June I
}

\begin{abstract}
We discuss recent research on the structure and particle acceleration properties of relativistic shock waves in which the magnetic field is transverse to the flow direction in the upstream medium, and whose composition is either pure electrons and positrons or primarily electrons and positrons with an admixture of heavy ions. Particlein-cell simulation techniques as well as analytic theory have been used to show that such shocks in pure pair plasmas are fully thermalized - the downstream particle spectra are relativistic Maxwellians at the temperature expected from the jump conditions. On the other hand, shocks containing heavy ions which are a minority constituent by number but which carry most of the energy density in the upstream medium do put $\sim 20 \%$ of the flow energy into a nonthermal population of pairs downstream, whose distribution in energy space is $N(E) \propto$ $E^{-2}$, where $N(E) d E$ is the number of particles with energy between $E$ and $E+d E$.

The mechanism of thermalization and particle acceleration is found to be synchrotron maser activity in the shock front, stimulated by the quasi-coherent gyration of the whole particle population as the plasma flowing into the shock reflects from the magnetic field in the shock front. The synchrotron maser modes radiated by the heavy ions are absorbed by the pairs at their (relativistic) cyclotron frequencies, allowing the maximum energy achievable by the pairs to be $\gamma_{ \pm} m_{ \pm} c^{2}=m_{i} c^{2} \gamma_{1} / Z_{i}$, where $\gamma_{1}$ is the Lorentz factor of the upstream flow and $Z_{i}$ is the atomic number of the ions. The shock's spatial structure is shown to contain a series of "overshoots" in the magnetic field, regions where the gyrating heavy ions compress the magnetic field to levels in excess of the eventual downstream value.

This shock model is applied to an interpretation of the structure of the inner regions of the Crab Nebula, in particular to the "wisps," surface brightness enhancements near the pulsar. We argue that these surface brightness enhancements are the regions of magnetic overshoot, which appear brighter because the small Larmor radius pairs are compressed and radiate more efficiently in the regions of more intense magnetic field. This interpretation suggests that the structure of the shock terminating the pulsar's wind in the Crab Nebula is spatially resolved, and allows one to measure $\gamma_{1}$ and a number of other properties of the pulsar's wind. We also discuss applications of the shock theory to the termination shocks of the winds from rotation-powered pulsars embedded in compact binaries. We show that this model adequately accounts for (and indeed predicted) the recently discovered X-ray flux from PSR 1957+20, and we discuss several other applications to other examples of these systems.
\end{abstract}

Subject headings: acceleration of particles - ISM: individual (Crab Nebula) - relativity — shock waves

\section{INTRODUCTION}

The excitation of diffuse, nonthermal astrophysical synchrotron sources by energy lost from central compact objects has long been a puzzle in high-energy astrophysics. Pulsars and their surrounding plerionic nebulae form the nearestat-hand and best-studied examples of this problem. Other examples include the excitation of extragalactic radio sources by jets, and some aspects of the emission from active galactic nuclei themselves. The Crab Nebula is the best studied of the

\footnotetext{
' Astronomy Department, Physics Department and Theoretical Astrophysics Center, University of California at Berkeley. Postal address: Astronomy Department, University of California, 601 Campbell Hall, Berkeley, CA 94720. E-mail: arons@astroplasma.berkeley.edu. Also associated with Institute of Geophysics and Plasma Physics and Plasma Physics Research Institute, Lawrence Livermore National Laboratory.

${ }^{2}$ Postal address: Physics Department, Jadwin Hall, Princeton University, Princeton, NJ 08544. E-mail: tavani@puppsr.princeton.edu.
}

plerions. It is typical of what we shall call "diffuse" plerions, in which synchrotron radiation and adiabatic expansion dominate the energy losses from relativistic electrons and positrons, and these losses are slower than the rate of the basic particle acceleration process. The termination of the outflow from a pulsar in a compact binary, as the relativistic wind encounters the mass lost from the companion star, may form an example of a "compact" plerion, the difference from the diffuse case being in the competition between radiation losses and the acceleration process itself.

Relativistic, magnetohydrodynamic (MHD) models of the coupling between pulsar and nebula are the most successful at the present time. Building on earlier ideas (Piddington 1957; Rees \& Gunn 1974; Kundt \& Krotscheck 1980), Kennel \& Coroniti ( $1984 \mathrm{a}, \mathrm{b})$ showed that such a wind from the pulsar, terminated by a standing, transverse magnetosonic shock wave, could give a good account of the nebular dynamics and 
of the nebular spectrum of high-energy photons (near-infrared, optical, X-rays and gamma rays), which are an instantaneous probe of the pulsar's outflow. The prominent results of their model are several. (1) The postshock flow fits into the slowly expanding nebula only if the upstream flow is almost entirely dominated by kinetic energy, with

$$
\sigma \equiv \frac{B_{1}^{2}}{4 \pi m N_{1} \gamma_{1} c^{2}} \ll 1
$$

a requirement first noticed by Rees \& Gunn (1974) in their mixed wind-vacuum wave model. Here $B_{1}$ is the upstream magnetic field, $N_{1}$ is the upstream density, and $\gamma_{1}$ is the Lorentz factor of the flow. (2) If the upstream flow is entirely composed of pairs (an assumption), and if the postshock energy distributions are power laws with $N(E) \propto E^{-s}$ (another assumption), then the synchrotron emission from the downstream flow can be fitted to the observed X-and $\gamma$-ray spectra of the Nebula, taken as an unresolved object, if $s \approx 2.3$. Once this fit is made, the optical and near-infrared spectra of the nebula (again taken as an unresolved object) are predicted (more or less) correctly by the model.

Subsequently, other authors have found that $\sigma \ll 1$ to be required by more elaborate dynamical and spectral considerations. Emmering \& Chevalier (1987) incorporated the time dependence introduced by nebular expansion into the dynamical model, and found $\sigma \approx 10^{-3}$ is required in order to match the observed rate of expansion, within the context of a spherical model of the nebula. By contrast, Kennel \& Coroniti's steady flow model yields $\sigma \approx 3 \times 10^{-3}$. Begelman \& Li ( 1992 , 1993 ) introduced the first nonspherical MHD model for the nonrelativistic expansion of the nebula, and found $\sigma \approx 3 \times$ $10^{-3}$ gives the best approximation to the ellipsoidal shape of the nebula. Their model neglects the interaction between the expanding "bubble" of relativistic particles and fields and the embedded filaments of nonrelativistic gas from which the optical emission lines arise. On the spectral front, de Jager \& Harding (1992) showed that if one adopts the magnetic field profile of Kennel \& Coroniti's ( $1984 \mathrm{a}$ ) $\sigma \approx 3 \times 10^{-3}$ model, one can construct a successful phenomenological model of the hard photon emission from the nebula, at photon energies from $\mathrm{MeV}$ through $\mathrm{GeV}$ to $\mathrm{TeV}$, based on synchrotron emission at photon energies up to a $\mathrm{GeV}$ and inverse Compton emission from the same population of relativistic electrons at photon energies above a GeV.

This theory is sufficiently successful as a macroscopic explanation of the phenomenology to provide a setting for investigation of the microscopic physics. Several questions are implied by the macroscopic flow model. Does a relativistic, magnetosonic shock in a pair plasma actually create the assumed power-law distributions downstream? If so, what is the mechanism of acceleration in such shocks? Are there other observable consequences of this mechanism, other than the desired particle acceleration, which allow one to test the shock hypothesis directly? We emphasize that one cannot appeal to the ever popular ideas of diffusive shock acceleration proposed for particle acceleration by interplanetary and nonrelativistic interstellar shocks (Bell 1978; Quenby \& Lieu 1989) even as extended to relativistic shocks with magnetic field parallel to the flow (e.g., Ellison, Jones, \& Reynolds 1990), since the termination shocks of pulsar winds must be fairly close to having the magnetic field perpendicular to the flow upstream when the plerion surrounds the pulsar-indeed, for the Crab, a laminar Archimedean spiral in the outflow from the pulsar would have $\Theta_{B n}$, the angle between the flow direction and the magnetic field in the shock frame where we observe the system, differing from $90^{\circ}$ only by one part in $10^{9}$ ! In addition, in relativistic shocks, the particles can stream ahead of the shock and participate in the back and forth motion across the front as required in diffusive shock acceleration only when $\Theta_{\mathrm{Bn}}^{\prime} \ll 1 / \gamma_{1}$, where $\Theta_{\mathrm{Bn}}^{\prime}$ is the angle between the flow direction and the magnetic field in the proper frame of the upstream fluid (Begelman \& Kirk 1990; Gallant et al. 1992). We will argue that $\gamma_{1} \sim 10^{6}$ in the Crab Nebula. Then the magnetic field is still almost exactly transverse even in the upstream fluid frame. Therefore, some other mechanism of shock acceleration must apply.

In relativistic shocks, the mechanisms of high-energy particle acceleration and of basic thermalization can be one and the same-accelerating relativistic particles can be part of the problem of shock structure itself. In fact, the highest energy electrons and positrons in the shock theory we outline below are those which have Larmor radii comparable to the gyroradii of the heavy ions which control the shock thickness, and are precisely the $e^{ \pm}$which give rise to the highest energy synchrotron photons observed in the nebular spectrum. Thus, the problems of shock structure and of nonthermal particle acceleration are one and the same.

\section{SHOCK STRUCTURE AND PARTICLE ACCELERATION}

Pulsar outflows must have extremely high Mach number. The Alfvén Mach number in the flow is $M_{\mathrm{A}}=\beta_{1} \gamma_{1} / V_{\sigma} \cong 10^{7}$ ! Under these circumstances, magnetic reflection of particles from the shock front plays an essential role in the shock dynamics. Analytically minded theorists traditionally have modeled collisionless shock waves by studying dissipation processes which occur in idealized solitary waves propagating in the dissipation free plasma. However, relativistic, magnetosonic solitary waves with unidirectional flow exist only for $M_{\mathrm{A}}<1+\left(1 / \gamma_{1}\right)($ Kennel \& Pellat 1976; Alsop \& Arons 1988; Chiueh 1989). At higher Mach number, incoming particles reflect from the enhanced magnetic field in the wave front and are set into Larmor gyration, leading to a new kind of magnetosonic solitary wave with reflected particles self-consistently incorporated (Alsop \& Arons 1988). This reflection process requires all the momentum of the incoming plasma to be temporarily stored in a magnetic overshoot, with $B_{\text {peak }} / B_{1}=(1+$ $\left.\sigma^{-1}\right)^{1 / 2}$ in the wave frame of a solitary wave in a pair plasma. The dependence of the overshoot on $\sigma$ alone is characteristic of relativistic magnetosonic shocks-once $\gamma_{1} \gg 1$, the dependence on $\gamma_{1}$ itself is entirely subsumed in the scale of the flow being that of the Larmor radius based on the upstream parameters.

While it is possible to construct solitary wave models with magnetically reflected particles in pair plasmas (Alsop \& Arons 1988), unraveling the instabilities and thermalization mechanisms in this highly inhomogeneous environment is a formidable task for analytical theory. We have made use of fully nonlinear, fully self-consistent particle-in-cell numerical 
simulations in order to uncover "experimentally" the relevant physics. The basic method is to integrate the equations of motion of many charged particles in their self-consistent fields (Langdon \& Lasinski 1976; Birdsall \& Langdon 1985). Suppose at some time $t_{n}$, one knows the electromagnetic field on a spatial grid, and one knows the positions and momenta of all the particles $\left\{\boldsymbol{x}_{j}\left(t_{n}\right), \boldsymbol{p}_{j}\left(t_{n}\right)\right\}$ (they are not on the grid). One then finds the Lorentz force at each particle's position and uses Newton's law (in relativistic form) to advance the particles to new phase space positions $\left\{\boldsymbol{x}_{j}\left(t_{n}+d t\right), \boldsymbol{p}_{j}\left(t_{n}+d t\right)\right\}$. One then calculates new fields at time $t_{n}+(d t / 2)$ using the charge densities and currents at time $t_{n}$, with the particles distributed on the grid with a suitable weight function. These new fields are used to advance the positions of the particles once again, with the time step set by various stability considerations.

We modeled shock waves in pair plasmas by setting up a one-dimensional spatial grid in the computer, with length anywhere from 10 to 40 Larmor radii based on the magnetic field and particle energy in the upstream flow. The initial magnetic field points exactly transverse to the flow velocity. At the initial time, the cold plasma fills the computational box, with flow Lorentz factor $\gamma_{1} \gg 1$ and a magnetic field polarized across the flow carried with the plasma at the fluid speed. The ratio of magnetic energy density to flow energy density upstream $\sigma$ is the only parameter of significance. As expected from theoretical considerations, simulations with the same value of $\sigma$ but differing $\gamma_{1}$ yield identical results, when $\gamma_{1} \gg 1$. We have surveyed parameter space with these spatially one-dimensional models from $\sigma=13$ down to $\sigma=0.001$.

At the injection point (the "left wall"), the particles are injected all with the same upstream velocity, and the magnetic field is carried with the fluid, by requiring the boundary electric field to be that of a perfectly conducting fluid. The opposite end of the grid is represented as a conducting rubber wallparticles bounce off the wall, which acts as a perfect conductor as far as the fields are concerned. It would be better to have outflow boundary conditions on the plasma and radiation, but so far a useful algorithm to implement these in the dense downstream plasma has not been constructed. Because the magnetic field points across the flow, the wall has no influence on the shock structure, once the point of particle reflection is more than a few Larmor radii away from the wall. In the low- $\sigma$ shocks of interest here, the wave energy is never more than a few percent of the thermal and kinetic energy of the plasma, so the conducting wall, which forces the downstream waves to be standing modes, has little influence on the shock once it its well upstream of the wall.

The details of the pair simulations are reported elsewhere (Langdon, Arons, \& Max 1988; Gallant et al. 1992). One finds an intense, quasi-coherent electromagnetic precursor traveling in front of the shock, while downstream, a few percent of the total energy is turned into a linearly polarized Rayleigh-Jeans spectrum of electromagnetic waves with temperature close to what one expects from the jump conditions. The incoming plasma stream is reflected from the shock front, followed by rapid thermalization of the Larmor gyration. The downstream spectra of electrons and positrons are almost perfect relativistic Maxwellians. Thus, transverse shocks in a pure pair plasma create a fully thermalized downstream medium, contrary to a basic assumption of the MHD model of plerionic supernova remnants. We emphasize that these simulations have more than enough resolution to include the effects of scattering of particles across the average magnetic field by long-wavelength electromagnetic fluctuations, if such modes were to form in the shock, a process suggested by Jokipii (1987) as the basis for diffusive Fermi acceleration in perpendicular shocks. Individual particles in the simulations do show "random" motions in the high-frequency fields formed within the shock front and radiated in the up- and downstream directions, with diffusion rates with respect to the background magnetic field comparable to the "Bohm" rate. However, their energy gains and losses are dominated by the wave emission and absorption discussed below.

We have done a few preliminary calculations with the magnetic field oblique to the flow, using what is otherwise the same one-dimensional model. So long as $\Theta_{B n}^{\prime}>50^{\circ}$, the results are semiquantitatively the same as for the purely transverse case, as one might suspect since the structure is dominated by the reflected particles and the rapid dissipation. Smaller obliquities may be different. We have also investigated some aspects of the two-dimensional structure of these shocks, motivated by the observation that the one-dimensional model leaves the plasma with a high temperature in the momenta orthogonal to the magnetic field, while it is still cold in the momenta along $B$. Such a medium is unstable, most prominently with respect to the Weibel instability. In one two-dimensional shock calculation, we have found this effect does heat the plasma in the direction along $B$, thus broadening the distribution of gyrational momenta, but the basic shock structure persists, with downstream anisotropy $T_{\perp} / T_{\|} \sim 2$ (Gallant et al. 1992).

The nature of the thermalization mechanism gives a clue to a shock model which does have nonthermal particle acceleration. As seen in the shock frame, the reflected pairs form gyrating rings with small momentum dispersion in the leading edge of the shock. Such rings are unstable-they form synchrotron masers (Zhelznyakov \& Suvorov 1972; Yoon 1990; Hoshino \& Arons 1991). Calculations of the growth rate for a homogeneous pair plasma, along with estimates of the maximum harmonic number that can be generated, suggest that the dissipation observed in the pure pair plasmas is due to the formation of synchrotron masers, with consequent plasma heating due to cyclotron absorption of the maser-generated extraordinary modes. Since the pairs collectively emit and reabsorb the randomly phased radiation at a rate rapid compared to the rate of flow through the shock front, it is no surprise that the radiation and the matter thermalize even though two-body Coulomb encounters are negligible.

Now imagine what happens if the upstream flow contains heavy ions, as well as pairs. Since all the species flow into the shock with the same speed (the upstream Larmor radii are small compared to the overall flow scale in which the shock is embedded), $Z N_{1 i}+N_{1+}=N_{1-}$. The ions have a rest mass large compared to that of the $e^{ \pm}$. Therefore, they will contribute the dominant kinetic energy in the flow, if $N_{1 i}>\left(m_{ \pm} /\right.$ $\left.m_{i}\right)\left(N_{1+}+N_{1-}\right)$. As the plasma encounters the shock, the lighter pairs, which have Larmor radii a factor $Z m_{ \pm} / m_{i}$ smaller than the ions, form a leading leptonic shock almost identical to the shock found in a symmetric plasma, if $N_{1 i} \ll$ $\left(N_{1+}+N_{1-}\right)$. The more massive ions cannot immediately respond to the change in plasma state induced by the pair shock, 
since the pair shock develops on the pair cyclotron time. The ions plow on through until they too are magnetically reflected in the enhanced magnetic field of the shock front. An electrostatic field in the direction of flow forms due to the charge imbalance created by the compression of the pairs with no immediate compression of the ions, with the electrostatic potential having magnitude $Z e \Phi \sim m_{i} \gamma_{1} c^{2}$. Once the ions are set into gyration by the reflection from the shock front, they too are synchrotron maser unstable, as they gyrate in the much denser background of shock heated pairs. However, the ion maser waves are preferentially absorbed by the more mobile pairs, rather than by the ions themselves. Thus, the pairs are in good contact with an "external" source of free energy, in the form of electromagnetic fluctuations extending down in frequency all the way to the ion cyclotron frequency based on upstream parameters. As a positron or electron cyclotron absorbs the high-frequency waves in the ion maser spectrum, it gains energy, lowers its cyclotron frequency, absorbs lower frequency modes, etc., until its cyclotron frequency matches that of the heavy ions, which occurs only when the energy of a lepton equals the incoming flow energy of a heavy ion, that is, when $\gamma_{e^{ \pm}}=\left(m_{i} / Z m_{ \pm}\right) \gamma_{1}$. This transfer of energy to the pairs has the consequence that the ions may only slowly thermalize, preserving their quasi-coherent Larmor gyration to substantial downstream distances.

Simulations of shock flows with ions (Hoshino et al. 1992) support these conjectures. In Figure 1 we show the electromagnetic structure from a simulation done in the same manner as the simulations of the purely leptonic shocks, but now containing ions with a charge to mass ratio appropriate to protons, at
$12 \omega_{c i}^{-1}$ after the plasma bounced off the simulation wall. In order to keep the computer time finite, the mass ratio is small, $m_{i} / m_{ \pm}=20$, and the density ratio is $N_{1 i} /\left(N_{1+}+N_{1-}\right)=0.2$, chosen as a compromise to have $N_{1 i} \ll\left(N_{1+}+N_{1-}\right)$ but still have most of the kinetic energy density in the upstream flow. In this case, $80 \%$ of the plasma flow energy is in the ions. The upstream flow is almost entirely dominated by the plasma kinetic energy flux- $\sigma=0.005$. While the choice of $\gamma_{1}$ matters only to the relative scale of the flow, in the interests of making the problem be of direct relevance to plerions and pulsars, we chose $\gamma_{1}=10^{6}$. Note the large overshoot in the magnetic field and the long wavelength oscillations downstream. The first overshoot occurs because as the ions reflect from the shock front, their flow momentum is stored in an increase in the magnetic pressure and in the thermal pressure of the pairs. Behind the overshoot, the ions continue gyrating quasi-coherently, creating compressional enhancements at each turning point, until their gyration finally thermalizes. These oscillations are spaced roughly one ion Larmor radius apart.

In Figure 2 we show zoomed views of the $p_{x}-x$ projections of ion phase space for a region of the simulation near the shock front itself, at 12 and 13 ion cyclotron times after the simulation began. One sees the quasi-coherent reflection of the ions, followed by their rapid thermalization. The figure also shows the unsteady character of the shock structure. On this scale, the structure of the pair shock, with its initial thermalization to Maxwellian distributions, is infinitesimal, achieved as the ions have barely begun to gyrate. Figure 3 shows the spectra of the downstream electrons and positrons. The positrons are no longer everywhere Maxwellian in energy space, but now have a
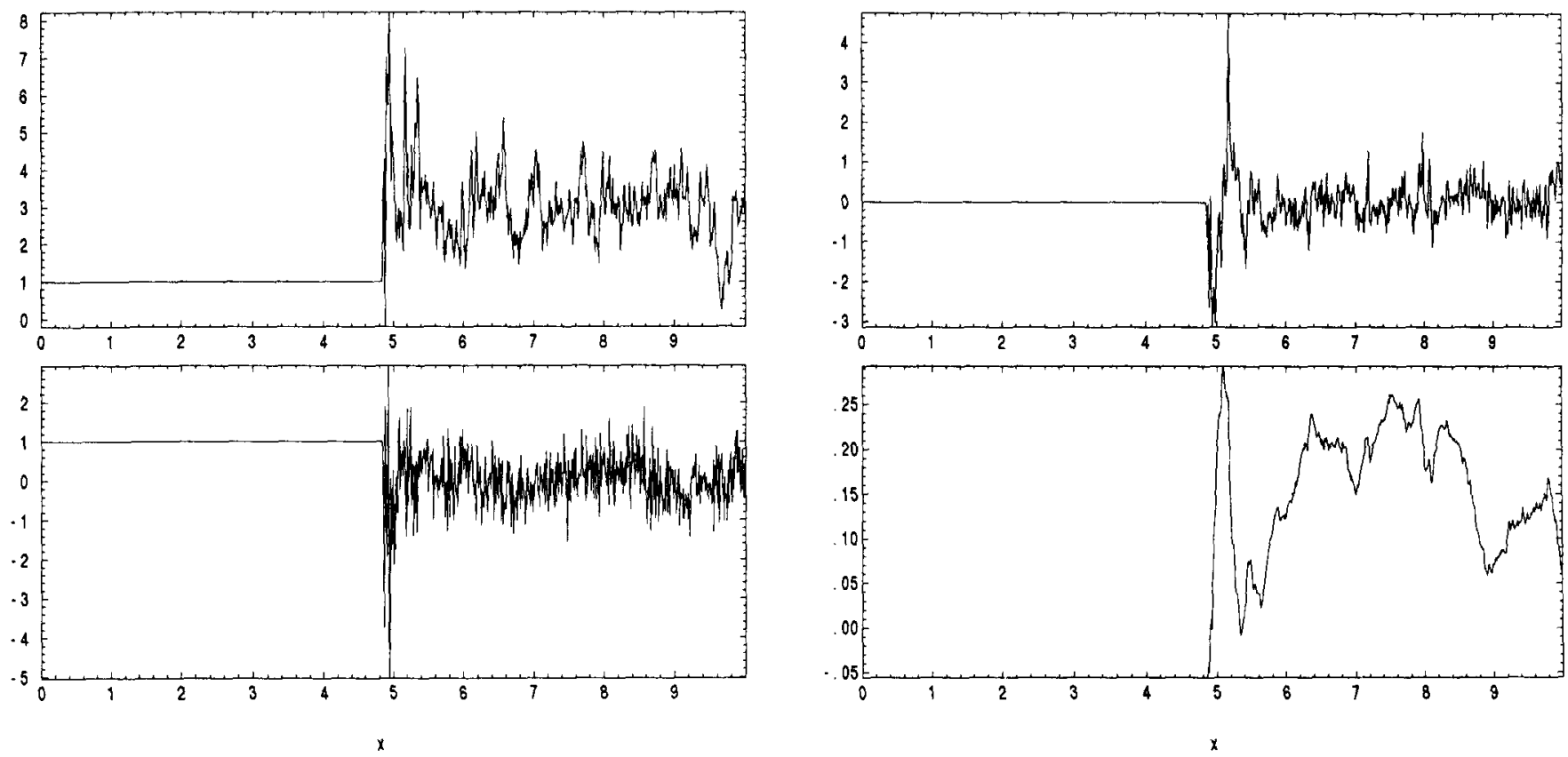

FIG. 1.-Electromagnetic structure of a relativistic magnetosonic shock in an electron-positron-proton plasma, at a time when the shock has crossed $50 \%$ of the simulation grid. Upper left panel: Magnetic field $B_{z}$, with upstream field amplitude equal to unity. Lower left panel: Transverse electric field $E_{y}$, with $E_{y}=\left(v_{1} / c\right) B_{z}$ at the injection wall. Upper right panel: Electrostatic field $E_{x}$. The downstream waves have electrostatic fields with amplitudes roughly one-half the magnitude of the electromagnetic field components, indicating strong elliptical polarization of the postshock extraordinary modes. Lower right panel: Electrostatic potential in units of $\gamma_{1} m_{i} c^{2} / e$. Since the potential never exceeds 0.3 , the electrostatic field in the shock front acts to decelerate the ion stream, but the reflection is magnetic, as in the shocks in pure pair plasmas. The unit of length is the ion Larmor radius based on upstream parameters. 

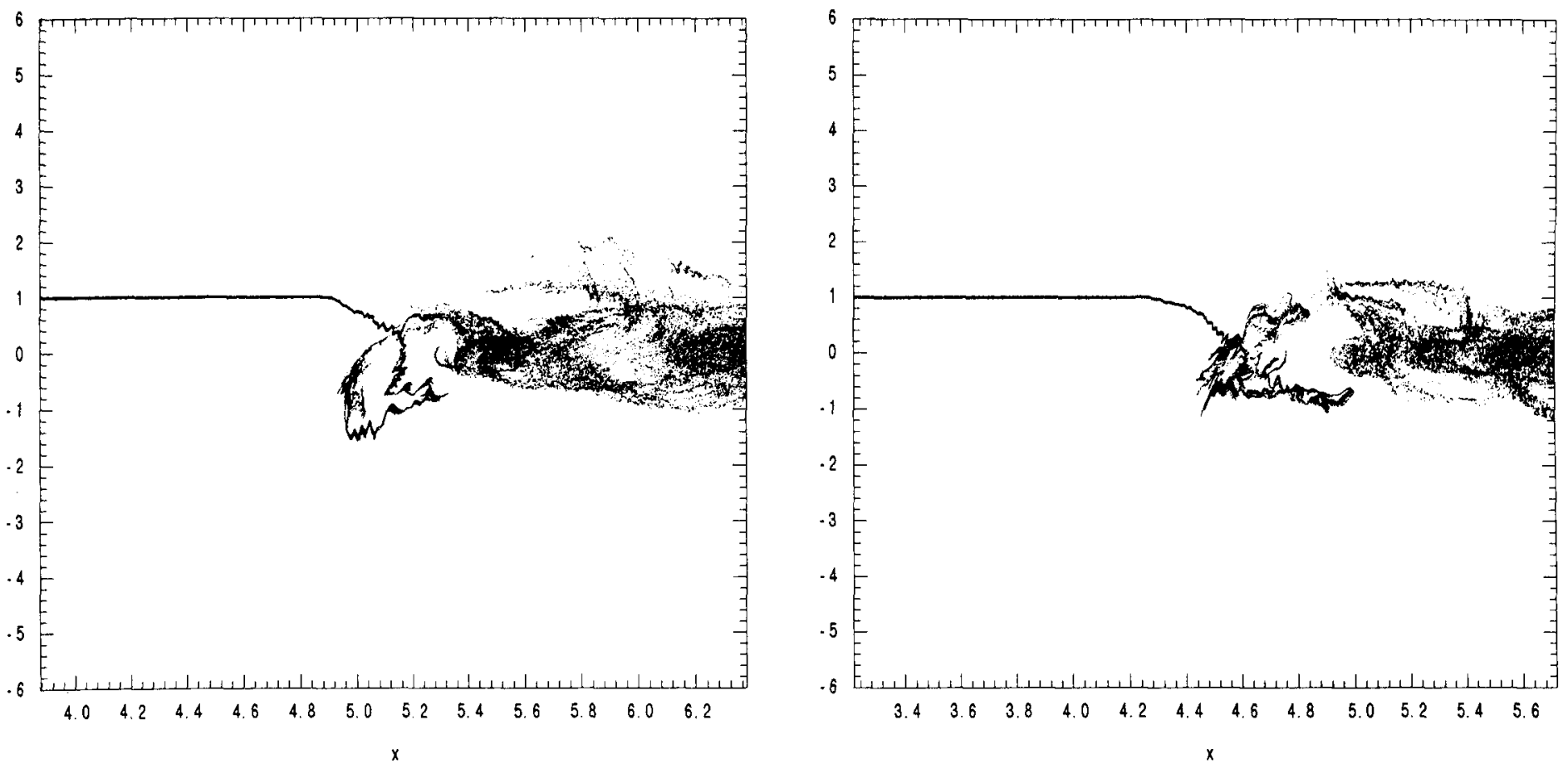

FIG. 2.-Ion phase space near the shock front. Left panel: Flow momentum in units of $10^{6} m_{i} c$ vs. $x$ for ions near the shock front at $t=12 / \omega_{c i 1}$. The unit of length is the ion Larmor radius based on upstream parameters. Note the initial deceleration of the ion stream in the electrostatic field, followed by the rapidly dissolving loop in the magnetic field. The main body of an ion Maxwellian distribution is produced within 2-3 Larmor times after the ions begin decelerating in the shock front. Right panel: ion flow momentum versus $x$ at $t=13 / \omega_{c i 1}$ near the shock front. These snapshots illustrate the unsteady character of the shock.

power-law distribution with $N(E) \propto E^{-s}$ with $s \approx 2$ at energy/ particle exceeding $\gamma_{1} m_{ \pm} c^{2}$ and have energy density comparable to $\sim 20 \%$ of the ions' kinetic energy density in the upstream flow. In this simulation, the electrons also show some sign of power-law behavior at energies exceeding $\gamma_{1} m_{ \pm} c^{2}$.

The positron spectrum is Maxwellian at energies below

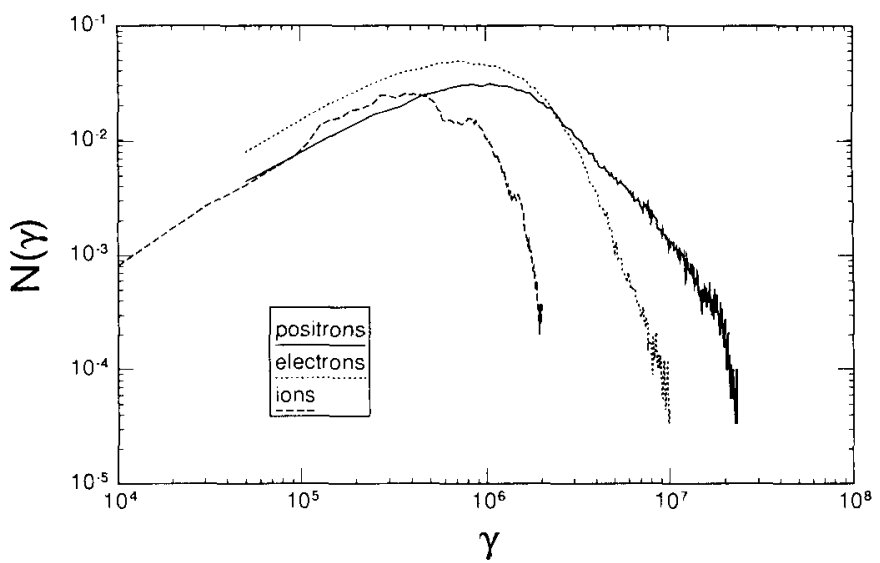

FIG. 3.-Postshock distributions. The positrons have a high-energy power-law spectrum with slope $s \approx 2$, while the electrons show evidence for a power-law suprathermal distribution with slope $s \approx 3.7$. The upper cutoff occurs where the energy/particle of the pairs equals the upstream energy/particle of the ions, as is expected from cyclotron resonant absorption of extraordinary modes emitted at and above the upstream ion cyclotron frequency. $\gamma_{1} m_{ \pm} c^{2}$, and has upper cutoff at positron energy equal to that of the upstream energy/particle of the protons, $\gamma_{1} m_{i} c^{2}$. Thus, a relativistic, low- $\sigma$ magnetosonic shock, composed of ions, electrons and positrons, with $N_{1 i} \ll N_{1+}+N_{1-}$ but with the upstream flow energy dominated by the ions, can accelerate the downstream positrons into a power-law distribution with high efficiency, with the slope of the power law being very close to what is desired for the macroscopic model. This particular simulation is highly resolved, with 64 particles per grid cell per species in the initial state. In other calculations of the shock structure with various values of $\sigma$ and of $\gamma_{1}$, and in simulations of the synchrotron maser process in isolation in a uniform medium, we have found the slope to be be of order 2. Figure 4 shows the spectral slopes found as a function of $\sigma$. For $\sigma=$ $0.0034,0.005$, simulations were done for a variety of numerical parameters ( number of particles/cell, number of grid cells per ion Larmor radius, etc.). The dispersion in the values of $s$ obtained defines the "error" in these numerical experiments in determining the form of the high-energy tail of the distribution function. The relatively small mass ratios and the upper limits to the number of particles per cell form the main sources of the variability in $s$ found in the various simulations with the same physical parameters. Figure 4 also shows the fraction of the downstream energy density contained in the nonthermal part of the particle spectra. For all the simulations, this efficiency is between $10 \%$ and $20 \%$, with the latter being the value appropriate to the physical conditions inferred in the Crab Nebula.

The precursor does not appear in this simulation, since the grid scale was equal to $0.2 c / \omega_{c 1+}$ based on upstream parame- 


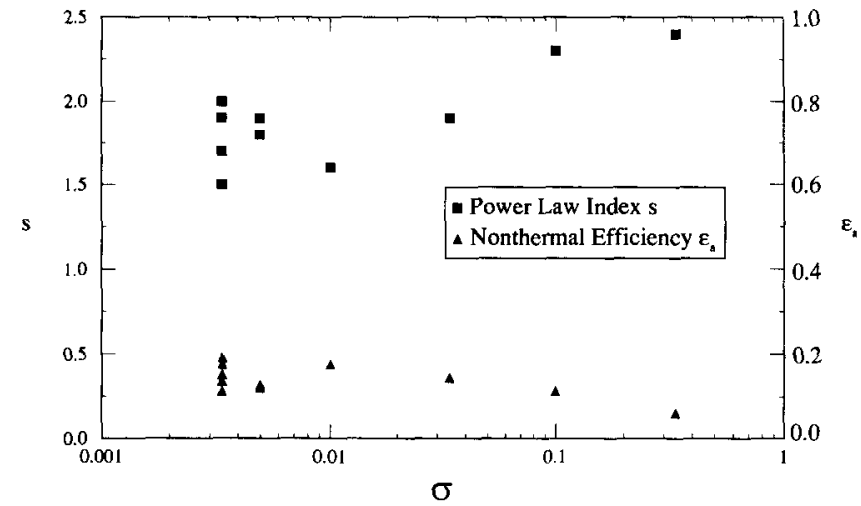

FIG. 4.-Nonthermal spectral index and acceleration efficiency as a function of $\sigma$. Squares show the the index $s$ (left ordinate axis) of the power-law part of the downstream positron spectra, as a function of the total $\sigma$ of the upstream electron-positron-proton flow. The triangles show the nonthermal particle acceleration efficiency of these shocks ( right ordinate axis), defined as the ratio of the downstream energy in the nonthermal part of the particle spectrum to the total downstream energy density.

ters, which leaves the precursor unresolved; in separate calculations we have shown that omitting the precursor does not affect the downstream particle acceleration. The electrostatic field in Figure 1 shows a strong double layer structure at the shock front, as the ions are first held back by the pairs frozen to the field, then begin gyrating and pull the pairs forward, while the potential $\Phi$ reaches $\sim 0.5 \gamma_{1} m_{i} c^{2} / e$ in the double layer region. Behind the shock, the magnetic field settles down to a mean value of $B_{2} \approx 3 B_{1}$, as one would expect from the MHD jump conditions for such a plasma-dominated flow, with intense extraordinary mode noise (Gallant et al. 1992). In the simulation frame, the average transverse electric field is noise with a mean of zero, while in the shock frame (only approximately well defined, since the shock velocity is not strictly steady around its mean value of $c / 3$ ), the mean transverse electric field is approximately constant.

It is likely that two-dimensional effects (primarily pitchangle scattering due to Weibel "turbulence," Yang et al. 1993a, b) will steepen the spectrum somewhat. In any case, it is clear that these electron-positron-ion shocks do produce downstream positron spectra whose characteristics are highly desirable for the modeling of plerions.

The physics of this acceleration is simple. In this nonsymmetric plasma, the extraordinary modes are no longer linearly polarized electromagnetic waves but have a noticeable electrostatic component as they propagate across the magnetic field. For frequencies between the relativistic analogue of the lower hybrid frequency and the relativistic cyclotron frequency of the heated pairs as they emerge from the leading leptonic shock, the ion extraordinary modes behave like magnetosonic waves in the pair plasma, and have elliptical polarization with the electric vector rotating in the left-handed sense with respect to the magnetic field. This is the same sense as the Larmor gyration of the positrons and of the ions. As a result, a positron can linearly absorb the magnetosonic modes at their cyclotron resonance (as well as at higher harmonics) and can gain energy. Because the polarization is elliptical, there is some power in right circularly polarized fields, so the electrons also can gain energy nonthermally from the ions. Because of the small mass ratio used in the simulations, the power in left-handed circularly polarized fields emitted by the ions is much larger than in right-handed circular polarization, so the positron acceleration in the simulations is much stronger than the acceleration of electrons. Under realistic conditions, quasi-linear theory suggests the acceleration of electrons and positrons will be much more comparable (Hoshino et al. 1992). It is possible to simulate this flow with mass ratios as high as 100 , which gives much larger dynamic range in the particle spectra and should show more comparable electron and positron acceleration; such simulations are in progress.

This mechanism works efficiently only if the collective synchrotron emission of the ions yields extraordinary mode frequencies greater than or equal to the thermal cyclotron frequency of the positrons just behind the leading leptonic subshock. The linear theory of the synchrotron maser instability in the ions (Hoshino \& Arons 1991) suggests that this is possible only if the upstream thermal momentum dispersion of the ions is small compared to $m_{ \pm} / m_{i}$. This is probably not a severe restriction, because the acceleration of the ions is likely to be by an electromagnetic mechanism, with the ion energy essentially unique on each streamline. In any case, adiabatic expansion in the outflow reduces any initial thermal dispersion drastically by the time the shock is reached. To the degree this shock acceleration model works successfully, this constraint on the acoustic Mach number of the flow should be regarded as a restriction on any theory of the wind. All of the simulations easily satisfy this requirement.

\section{APPLICATION TO PLERIONS}

\subsection{The Wisps in the Crab Nebula as Resolved Shock Structure}

One can get the flavor of models based on these results from the following simple considerations. Suppose the magnetosphere of the Crab pulsar emits a dense flow of pairs, with $\dot{N}_{ \pm} \sim 10^{38} \dot{N}_{ \pm, 38}$ coming out in the broad sector around the rotational equator where the pulsar must emit the wind required to feed the torus of X-, and gamma-ray emission in the Nebula (Aschenbach \& Brinkmann 1975; Pelling et al. 1987). A number of magnetospheric models suggest such a flow is possible. In addition, suppose the magnetospheric electrodynamics causes an outflow of heavy ions to be pulled up from the stellar surface and accelerated to high energy in the outflow around the magnetic equator (Arons 1983). The ion flux is constrained by the electrodynamics to be close to the Goldreich-Julian flux, $\dot{N}_{\mathrm{GJ}}=2 \Omega^{2} \mu / Z e c \approx 2 \times 10^{34} / Z$ for this pulsar, where $Z$ is the charge on the ions. The resulting ratio of the mass densities in the wind is

$$
\rho_{i} \equiv \frac{m_{i} N_{i 1}}{m_{ \pm}\left(N_{1+}+N_{1-}\right)}=918 \frac{A}{Z} \frac{\Omega^{2} \mu}{e c \dot{N}_{ \pm}}
$$

where $A$ is the atomic number of the ions, $\mu$ is the pulsar's magnetic moment, and $\Omega$ is its angular velocity. Solving for $Z$ yields $Z \approx 3(A / 56)\left(1 / \dot{N}_{ \pm, 38}\right)\left(\rho_{i} / 2\right)$ in the case of the Crab. More refined analysis (Gallant \& Arons 1993) suggests the charge on the ions as they are extracted from the stellar surface is comparable to the ions' charge as they enter the shock structure in the Nebula, that they are fairly close to being fully 
stripped, and that helium is the most likely candidate for the ion composition.

These ions might be accelerated through a fair fraction of the total magnetopsheric potential $\Phi_{\text {mag }} \approx \Omega^{2} \mu / c^{2} \sim 5 \times 10^{16} \mathrm{~V}$, corresponding to $\gamma_{1} \sim 5 \times 10^{7}(Z / A)\left(\Phi / \Phi_{\text {mag }}\right)$. If the shock puts $\sim 20 \%$ of the outflow energy into the accelerated pairs, as our simulations suggest, and if these pairs radiate essentially all of this in X-rays and gamma rays, as is indicated by the macroscopic flow models, then one would conclude, from the hard photon Iuminosity of the nebula, that the ions experience an acceleration potential $\Phi / \Phi_{\text {mag }} \sim 0.2 / I_{45}$, where $I_{45}$ is the moment of inertia of the neutron star in units of $10^{45} \mathrm{cgs}$.

We can go much further. From momentum conservation, one expects the shock to occur roughly $10^{17.5} \mathrm{~cm}$ from the pulsar (Rees \& Gunn 1974). Optical observations, schematically illustrated in Figure 5, reveal the presence of the curious "wisps" in the surface brightness in this region, a series of enhancements in the surface brightness elongated in the direction perpendicular to the flow lying to the northwest of the pulsar; there is also a fainter wisp to the southeast (Scargle 1969; van den Bergh \& Pritchet 1989). The broad-band synchrotron emissivity in the magnetic overshoot and downstream magnetic compressions shown in Figure 1 is readily found to be proportional to the $B^{11 / 3}$, assuming the downstream pitch-angle scattering has largely isotropized the pairs; if the pairs are not well isotropized but gyrate predominatly with their momenta largely orthogonal to the toroidal magnetic field, as turns out to yield a better model of the wisps' surface brightness (Gallant \& Arons 1993), the bolometric emissivity is proportional to $B^{4}$. The essential new astrophysical idea is to iden- tify the enhanced surface brightness expected in these compressions with the observed wisps.

From this identification, one can extract a host of conclusions. The details of these will be presented elsewhere. However, several qualitative statements can be made immediately. (1) A reasonable model, with $\sim 10^{37.6}$ pairs s $^{-1}$ flowing out in the wind and $\sim 6 \times 10^{34} / Z$ ions $s^{-1}$ with charge $Z \sim 2$ yields a roughly correct optical surface brightness for the thin wisp and wisp 1 and for the thickness of wisp 1 itself, if $\gamma_{1} \sim 5 \times 10^{6}$ (this value for $\gamma_{1}$ assumes an ion charge to mass ratio appropriate to fully ionized helium, or heavier fully ionized ions). (2) The series of downstream, large amplitude compressions of the plasma are spaced roughly one ion Larmor radius apart. This spacing is similar to that seen between the first few wisps in van den Bergh \& Pritchett's (1989) image if $\gamma_{1} \sim 5 \times 10^{6}$, the same value as leads to a proper representation of wisp 1's thickness.

For these parameters, the Larmor radius of the heavy ions based on upstream parameters is comparable to the radius itself, where the shock forms in the Nebula. Therefore, the quasi-spherical divergence of the flow must be incorporated in the model, if any quantitative comparison to observations is to be made. In Figure 6 we show the structure of the magnetic field including the spherical divergence and the effects of charge separation between the ions and the pairs, but omitting the dispersion in the ion momenta created by the ion synchrotron maser instability (Gallant, Arons, \& Langdon 1993; Gallant \& Arons 1993). Since the surface brightness varies as a high power of the magnetic field strength (see above), spherical divergence alone already explains much of the rapidly de-

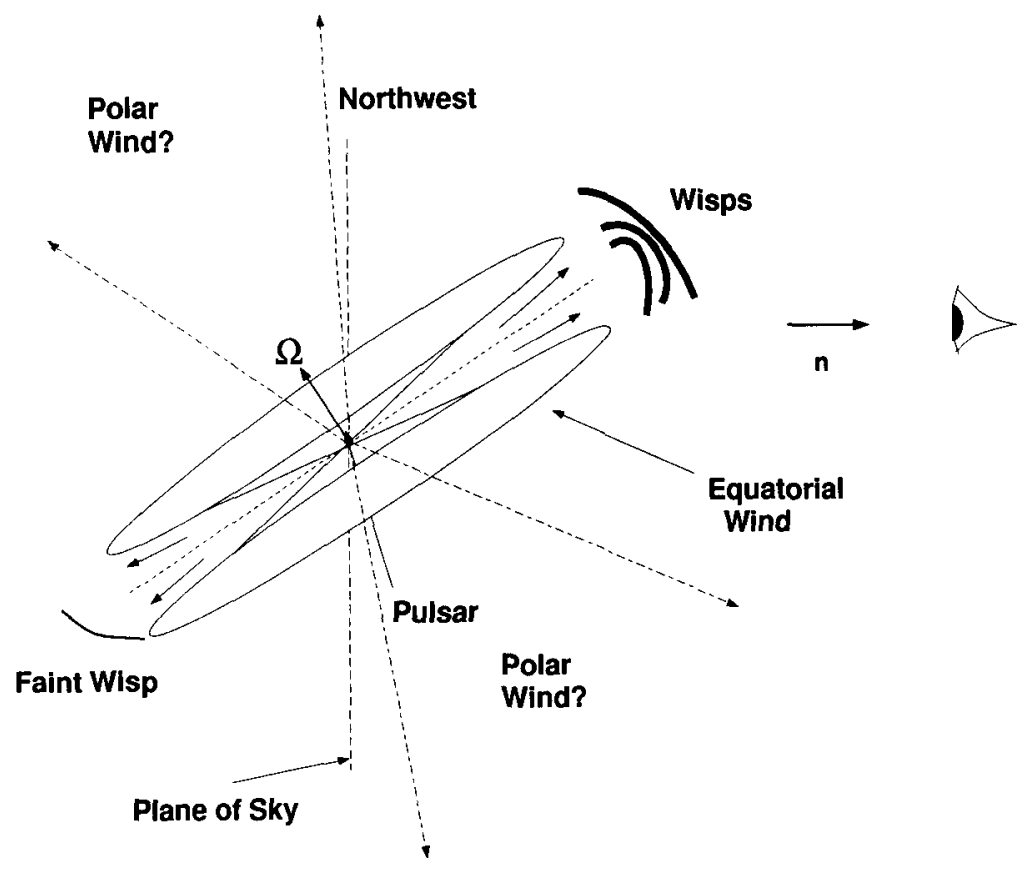

FIG. 5.-Representation of the termination region of the relativistic wind lying in the rotational equator of the Crab pulsar. The wind's magnetic field is toroidal with respect to the pulsar's rotation axis; therefore, it is transverse to the line of sight which intercepts the pulsar. The wisps are identified with the regions of magnetic compression within the shock structure, where the volume synchrotron emissivity is enhanced. The faint wisp has lower surface brightness because of the relative Doppler boost of the intensity between flow approaching and receding from the observer. The wisps appear as arcs rather than rings because the radiating pairs' momenta are not isotropically distributed with respect to the magnetic field direction, but instead are predominantly transverse to $B$. Therefore, emission is observable only from the arcs of the toroidal field which are close to being transverse to the line of sight. 


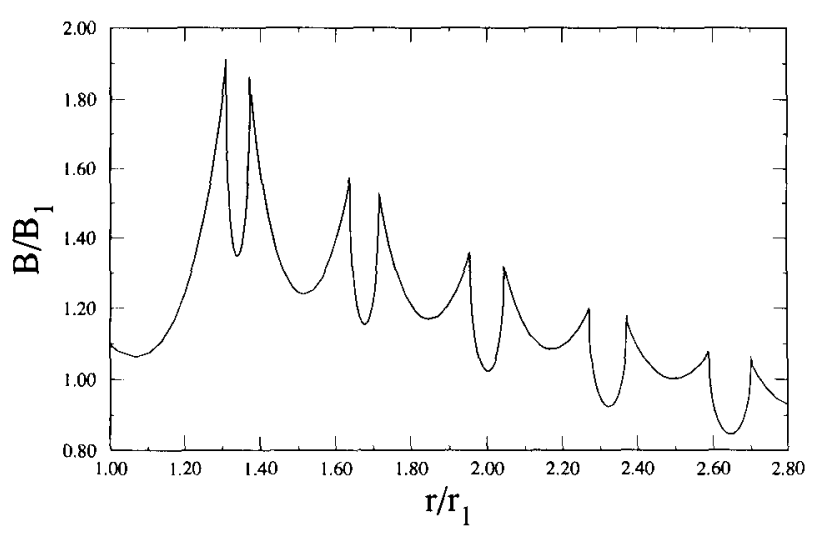

Fig. 6.-Magnetic structure of an adiabatic solitary wave model of the wisps in the Crab Nebula. Spherical divergence in the flow is included, but the ions are assumed to reflect from the shock front without randomization of the momenta. The pairs are assumed to be an adiabatic MHD fluid, thermalized by the pair subshock at $r=r_{1}$. The succesive epicycles in the ion flow combined with spherical divergence compresses the magnetic field into the the series of "double horned" magnetic overshoots of declining amplitude; these are reflected in a series of surface brightness enhancements in the nebular emission.

clining amplitude of the wisps as a function of increasing distance from the pulsar. (3) The spectrum of wisp 1 should be close to that of Maxwellian synchrotron emission. The spectrum for a uniform medium is well known (Jones \& Hardee 1979). With $B \sim 10^{-4} \mathrm{G}$ and pair temperature $\sim 5 \times$ $10^{6} m_{ \pm} c^{2}$, the characteristic exponential decline of the spectrum from a relativistic Maxwellian should appear above $\sim 25$ eV. (4) Successive wisps further from the ion overshoot should show a progressively more nonthermal spectrum, as the ions transfer their energy to the pairs. (5) The flow velocity of the pairs throughout this region is between $0.3 c$ and $0.5 c$, which provides a Doppler asymmetry to the luminosity just about right to explain the brightness ratio seen between wisp 1 and the one faint wisp observable to the pulsar's southeast. Possible partial anisotropy of the pairs with respect to the local magnetic field enhances this conclusion. (6) The shocks observed in our simulations are unsteady, as is shown in Figure 2. Translated into the shock frame, which is where we observe the Crab Nebula, the unsteady shock velocity becomes either anharmonic oscillation of the position of the first ion overshoot (= wisp 1), or disappearance and reformation of wisp 1, on a timescale of several months, a phenomenon consistent with time variability of the wisps reported long ago by Scargle (1969). Such variability should be uncorrelated with pulsar glitches and other rotational anomalies. In addition, such unsteady behavior may imply unsteady nebular emission at energies exceeding $1 \mathrm{MeV}$, as has been occasionally reported. (7) The time variable overshoots can transfer further energy from the gyrating ions to the pairs in the downstream medium, over and above that found in the spatially one-dimensional simulations. Because of the finite rate of pitch-angle scattering present downstream, magnetic pumping can increase the amount of energy transferred to the pairs, and can also act to transform the Maxwellian low-energy pair spectra into nonthermal form, thus allowing the same shock model to explain both the highenergy photons and the radio synchrotron emission from plerions (Arons 1993). With this spatially two-dimensional effect appended to the theory, the parameters derived from the wisp model are appropriate to those expected in the diffuse X-ray source formed further downstream. Detailed modeling (Gallant \& Arons 1993) shows that these rough numerical conclusions stand up to careful analysis of the flow and predicted surface brightness distribution; in addition, the detailed work reveals that pitch-angle anisotropy in the pairs is an essential feature of the shock structure, which reveals its presence in the progressive broadening of the wisps with increasing distance downstream. (8) The maximum energy reached by pairs in the theoretical model of the shock structure is equal to the energy per particle of the ions in the upstream flow, $E_{\max \pm}=\gamma_{1} m_{i} c^{2} \approx$ $2 \times 10^{15} \mathrm{eV}$ when the ions are fully stripped helium (drawn electrically from the top of the neutron star's atmosphere). This upper cutoff of the accelerated pair distribution yields synchrotron photons whose spectrum cuts off exponentially at energies above $1 \mathrm{GeV}$, as found in de Jager \& Harding's (1992) phenomenological model. The details of the formation of the gamma-ray spectrum are somewhat different, however, since the magnetic structure both includes the strong overshoots introduced by the reflected ions, and also has an average profile differing from that used by de Jager and Harding because of the anisotropic character of the pairs' pressure.

Therefore, we suggest that the structure of a relativistic shock in a relativistic wind composed of pairs plus heavy ions has its spatial structure revealed to us in the detailed morphology of the wisp region in the Crab Nebula. That this may be so is a consequence of the very high rigidity of the outflow from the pulsar, a result in accord with the enormous voltages developed in the magnetosphere combined with the negligible radiation drag on the flow near this photon poor compact object.

\subsection{Compact Plerions: Pulsar Winds in Binaries}

The same ideas can be applied to the millisecond radio pulsars recently discovered in compact binaries (Fruchter, Stinebring, \& Taylor 1988), whose relativistic winds interact with the mass lost from the "normal" stellar companion (Kluzniak et al. 1988; Phinney et al. 1988; Ruderman, Shaham, \& Tavani 1989). The geometry of the flow within such a binary is shown in Figure 7. In the case of PSR $1957+20$, if one assumes the same fraction of the potential drop on open field lines goes into the energy / particle of this pulsar's wind as appears in the wind from the Crab pulsar, one expects $\gamma_{1} \sim 10^{5.7}$, while if $\sigma$ is as small as it appears to be in the Crab pulsar's wind, the magnetic field behind the termination shock of the relativistic wind is on the order of $10 \mathrm{G}$, since the shock occurs less than one solar radius from this pulsar, at least along the line of centers between the pulsar and the companion. In this strong field, synchrotron losses limit the energies of the accelerated pairs to values much less than $\gamma_{1} m_{i} c^{2}$, and convert almost all of the energy of the part of the pulsar's wind shocked within the binary into high-energy radiation. The pairs accelerate to energies on the order of $10^{6} m_{ \pm} c^{2}$, and up to $20 \%$ of the wind energy is given up as 1-10 MeV synchrotron photons, whose flux at Earth is expected to be $10^{-6}$ photons $/ \mathrm{cm}^{2} \mathrm{~s}$ (Arons \& Tavani 1993), about an order of magnitude too small to be detected by the OSSE and COMPTEL instruments on CGRO. The soft X-ray instruments on ROSAT are much more sensitive, and in spite of their narrower bandwidth, the X-ray emission from the shocked wind in the binary is predicted to yield a 


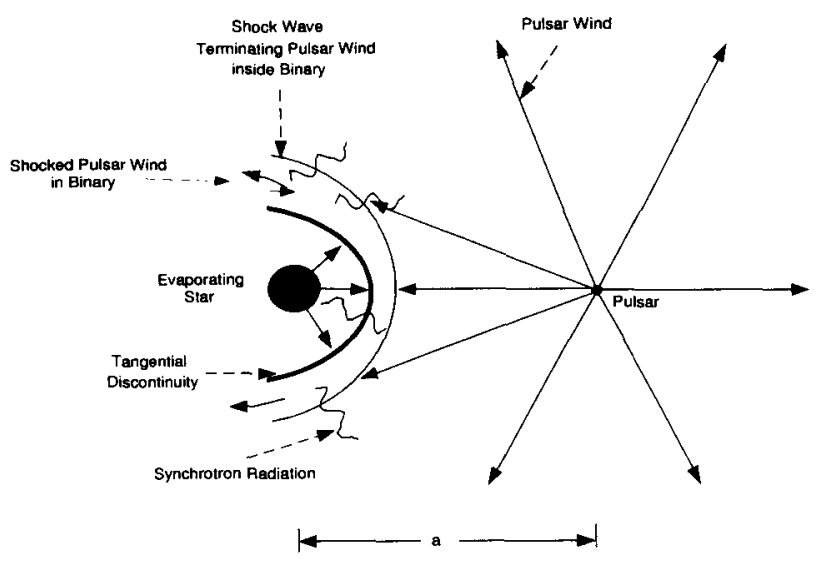

FIG. 7.-Schematic of the collision between the relativistic pulsar wind and the nonrelativistic mass lost from the companion in the PSR 1957+20 system. The scale is $a=1.7 \times 10^{11} \mathrm{~cm}$.

flux of approximately $2 \times 10^{-3}$ photons $\mathrm{s}^{-1}$ in the ROSAT band (Arons \& Tavani 1993). Indeed, simultaneously with this prediction, Fruchter et al. (1992) and Kulkarni et al. (1992) found just such a flux. The shocked wind in this binary forms a compact plerion, since the synchrotron losses now occur on a timescale competetive with the rate of acceleration of the pairs in the shock front, and not only with the rate of downstream flow, as is the case in the Crab Nebula.

The pulsar's companion intercepts at most $20 \%$ of the pulsar's wind. Most of the relativistic wind goes into blowing the nebular bubble observed in interstellar $\mathrm{H} \alpha$ emission around the binary (Kulkarni \& Hester 1988), but because of the much stronger magnetic field within the close confines of the binary, most of the high-energy emission photon comes from within the binary rather than from the nebular bubble-the relativis-

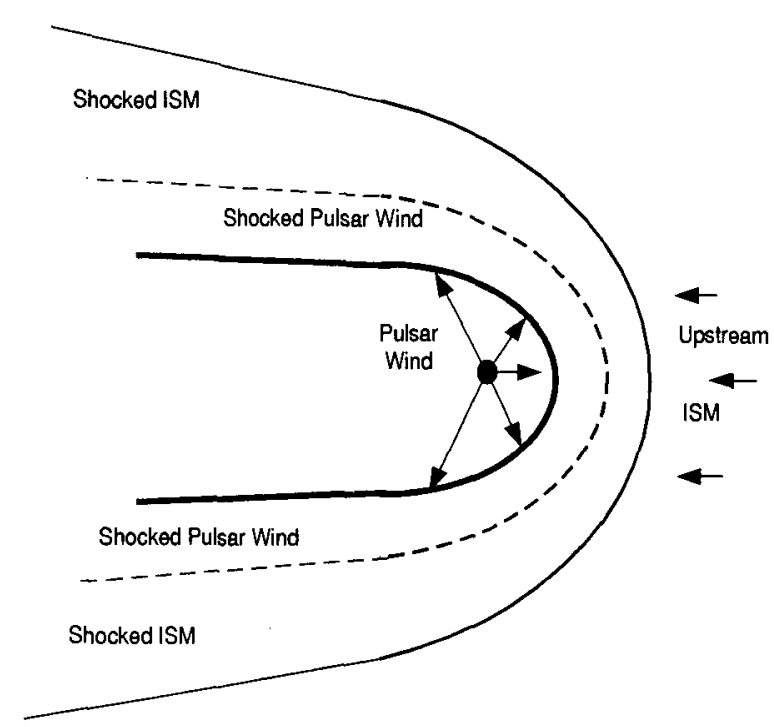

FIG. 8.-Schematic of the plerion blown by the pulsar wind from PSR 1957+20. The distance from the pulsar to the "nose" of the cometary nebula formed in the interstellar medium is about $2 \times 10^{16} \mathrm{~cm}$. The visible $\mathrm{H} \alpha$ nebula forms in the shocked interstellar medium. The shocked relativistic gas from the pulsar has not yet been observed. tic plasma in the nebular bubble is even more diffuse than that in the Crab Nebula. The geometry of the bubble is shown in Figure 8.

These ideas can be generalized into a broad classification of pulsar-driven nebulae, depending on the relative importance of the radiative losses in comparison to the adiabatic losses in the downstream flow and in comparison to the pair acceleration time in the shock front, where in general inverse Compton losses must be included as well as those due to synchrotron radiation. Perhaps the most intriguing application may be to the recently discovered binary pulsar PSR 1259-63 (Johnston et al. 1992) in a high-mass system, whose elliptic orbit (illustrated in Fig. 9) will carry the wind termination shock through a range of termination pressures as we watch it, and may sample the mass loss from the Be star at sufficiently high density to suppress completely the relativistic wind when the pulsar is near periastron. As a result, this system may be as informative as the Crab Nebula as a "laboratory" for diagnosing pulsar winds and their termination shocks.

Finally, we conclude with an intriguing speculation. The leading edge shock in the pairs emits a few percent of the flow energy in the pairs in the form of high brightness temperature extraordinary modes, whose spectrum extends at least to several hundred harmonics above the fundamental cyclotron frequency in the pairs (Gallant et al. 1992). These authors suggested that this high brightness temperature radiation might be the explanation of the apparent high brightness temperature centimeter wave radio emission recently observed in some active galactic nuclei (Wagner et al. 1990), since relativistic shocks are likely to form in the magnetic fields anchored to the accretion disks around the giant black holes believed to be in AGNs. Here we point out that the strong magnetic fields ( $\geq 10$ $\mathrm{G}$ ) in the termination shocks of the winds from pulsars within compact binaries might also lead to possibly observable collective radio emission, most likely at meter wavelengths. Low-frequency study of these systems, searching for unpulsed high brightness temperature emission, would be a worthwhile experiment.

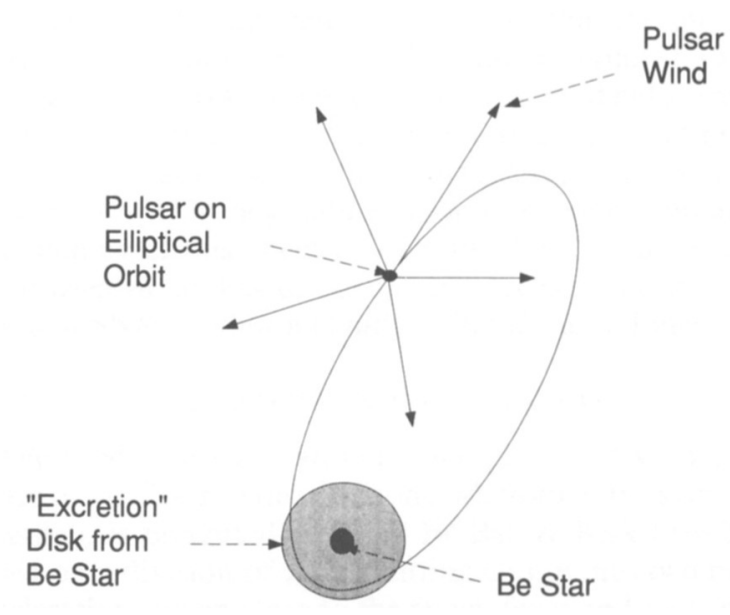

Fig. 9.- - Schematic of the binary system containing PSR 1259-63 on an elliptical orbit around its companion Be star. The collision of the pulsar wind with the excretion disk from the Be star can yield potentially observable X-rays, while if the pulsar dives deep enough into the excretion disk, the formation of the pulsar wind may be suppressed and the neutron star may make a transition to an accreting X-ray pulsar. 
The research described here on relativistic shock waves was supported in part by NSF grant AST-9115093 and by IGPPLLNL grant 92-23, both to the University of California at Berkeley. M.T. acknowledges support by NASA grant GRO/
PFP-91-23 to Princeton University. Part of the work was performed under the auspices of the U.S. Department of Energy at the Lawrence Livermore National Laboratory under contract W-7405-Eng-48.

\section{REFERENCES}

Alsop, D., \& Arons, J. 1988, Phys. Fluids, 31, 839

Arons, J. 1983, in Proc. Workshop on Electron-Positron Pairs in Astrophysics, ed. M. L. Burns, A. K. Harding, \& R. Ramaty (New York: AIP) , 163

Arons, J., \& Tavani, M. 1993, ApJ, 403, 249

Aschenbach, B., \& Brinkmann, W. 1975, A \& A, 41, 147

Begelman, M. C., \& Kirk, J. G. 1990, ApJ, 353, 66

Begelman, M. C., \& Li, Z.-Y. 1992, ApJ, 397, 187 . 1993, ApJ, submitted

Bell, A. R. 1978, MNRAS, 182, 147

Birdsall, C. K., \& Langdon, A. B. 1985, Plasma Physics via Computer Simulation (New York: McGraw-Hill)

Chiueh, T. 1989, Phys. Rev. Lett., 63, 113

de Jager, O. C., \& Harding, A. K. 1992, ApJ, 396, 161

Ellison, D. C., Jones, F. C., \& Reynolds, S. P. 1990, ApJ, 360, 702

Emmering, R. T., \& Chevalier, R. A. 1987, ApJ, 321, 334

Fruchter, A. S., Stinebrung, D. R., \& Taylor, J. H. 1988, Nature, 333, 237

Gallant, Y. A., \& Arons, J. 1993, ApJ, submitted

Gallant, Y. A., Arons, J., \& Langdon, A. B. 1993, in Physics of Isolated Pulsars, ed. K. van Riper, R. Epstein, \& C. Ho (Cambridge: Cambridge Univ. Press), in press

Gallant, Y. A., Hoshino, M., Langdon, A. B., Arons, J., \& Max, C. E. 1992, ApJ, 391, 73

Hoshino, M., \& Arons, J. 1991, Phys. Fluids B, 3, 818

Hoshino, M., Arons, J., Gallant, Y. A., \& Langdon, A. B. 1992, ApJ, 390, 454

Johnston, S., Manchester, R. N., Lyne, A. G., Bailes, M., Kaspi, V. M., Guojun, Q., \& D'Amico, N. 1992, ApJ, 387, L37

Jokipii, J. R. 1987, ApJ, 313, 842

Jones, T. W., \& Hardee, P. E. 1979, ApJ, 228, 268
Kennel, C. F., \& Coroniti, F. V. 1984a, ApJ, 283, 694

Kennel, C. F., \& Coroniti, F. V. 1984b, ApJ, 283, 710

Kennel, C. F., \& Pellat, R. 1976, J. Plasma Phys., 15, 335

Kluzniak, W., Ruderman, M. A., Shaham, J., \& Tavani, M. 1988, Nature, 334,225

Kulkarni, S. R., \& Hester, J. J. 1988, Nature, 335, 801

Kulkarni, S. R., Phinney, E. S., Evans, C. R., \& Hasinger, G. 1992, Nature, 359,300

Kundt, W., \& Krotscheck, E. 1980, A \& A, 83, 1

Langdon, A. B., Arons, J., \& Max, C. E. 1988, Phys. Rev. Lett., 61, 779

Langdon, A. B., \& Lasinski, B. F. 1976, Meth. Comput. Phys., 16, 327

Pacini, F. 1967, Nature, 216, 567

Pelling, R. M., Paciesas, W. S., Peterson, L. E., Makashima, K., Oda, M., Ogawara, Y., \& Miyamoto, S. 1987, ApJ, 319, 416

Phinney, E. S., Evans, C. R., Blandford, R. D., \& Kulkarni, S. R. 1988, Nature, 333, 832

Piddington, J. H. 1957, Aust. J. Phys., 10, 530

Quenby, J. R., \& Lieu, R. 1989, Nature, 342, 654

Rees, M. J., \& Gunn, J. E. 1974, MNRAS, 167, 1

Ruderman, M. A., Shaham, J., \& Tavani, M. 1989, ApJ, 336, 507

Scargle, J. D. 1969, ApJ, 156, 401

van den Bergh, S., \& Pritchet, C. J. 1989, ApJ, 343, L69

Wagner, S., Sanchez-Pons, F., Quirrenbach, A., \& Witzel, A. 1990, A \& A, 235, L1

Yang, T.-Y. B., Gallant, Y. A., Arons, J., \& Langdon, A. B. 1993a, in Physics of Isolated Pulsars, ed. K. van Riper, R. Epstein, \& C. Ho (Cambridge: Cambridge Univ. Press), in press . 1993b, Phys. Fluids, in press

Yoon, P. 1990, Phys. Fluids B, 3, 867

Zhelznyakov, V. V., \& Suvorov, E. V. 1972, Ap \& SS, 15, 24 